\title{
Why Factor Analysis Often is the Incorrect Model for Analyzing Bipolar Concepts, and What Model to Use Instead
}

\author{
Wijbrand H. van Schuur and Henk A. L. Kiers \\ University of Groningen, The Netherlands
}

\begin{abstract}
Factor analysis of data that conform to the unfolding model often results in an extra factor. This artificial extra factor is particularly important when data that conform to a bipolar unidimensional unfolding scale are factor analyzed. One bipolar dimension is expected, but two factors are found and often are interpreted as two unrelated dimensions. Although this extra factor phenomenon was pointed out in the early 1960 s, it still is not widely recognized. The extra factor phenomenon in the unidimensional case is reviewed here. A numerical illustration is provided, and a num ber of diagnostics that can be used to determine whether data conform to the unidimensional unfolding model better than to the factor model are discussed. These diagnostics then are applied to an empirical example. Index terms: factor analysis, factor interpretation problems, rating scales, unfolding diagnostics, unfolding model.
\end{abstract}

A technique widely used for data collection is to ask respondents to answer questions according to rating scale categories, which may range from agree very much to disagree very much. Rating scale data often are analyzed with factor analysis, which identifies variables that can be attributed to the same latent factor and provides the researcher with a measurement value-a factor score-for each individual on each of the factors.

The use of factor analysis has sometimes led to unexpected results. In particular, indicators that were meant to operationalize one bipolar dimension have been found, surprisingly, to represent two independent, unipolar factors. For example, in studies of mood state, self-descriptive adjectives like "happy"

APPLIED PSYCHOLOGICAL MEASUREMENT

Vol. 18, No. 2, June 1994, pp. 97-110

(C) Copyright 1994 Applied Psychological Measurement Inc. 0146-6216/94/020097-14\$1.95 and "sad" have not all loaded highly (but with opposite signs) on the same factor, but instead have loaded highly and positively on only one of two independent, unipolar factors (e.g., Diener \& Emmons, 1985; Green \& Goldfried, 1965; Lorr \& Shea, 1979; Nowlis \& Nowlis, 1956). Similarly, factor analysis of political data (e.g., agreement with political measures, preference for political leaders) has suggested, counter to expectation, that "liberalism" and "conservatism" are two independent, orthogonal factors rather than one bipolar factor (e.g., Conover \& Feldman, 1981; Krosnick \& Weisberg, 1988; Weisberg, 1980).

This paper explains that the identification of two factors, when one factor is expected, is an artifact caused by using factor analysis on data that would be more appropriately analyzed with a unidimensional unfolding model (Coombs \& Kao, 1960; Davison, 1977; Ross \& Cliff, 1964). The explanation does not originate here, but it is presented to emphasize its importance for social scientists, because the use of factor analysis for data that should be unfolded is still rather widespread.

\section{Factor Analysis and the Unfolding Model}

Stated very generally, the problem arises because unfoldable data violate fundamental assumptions of the factor analysis model. Factor analysis assumes that values of the observed variables are linearly (or even monotonically) related to values on the underlying latent variables; however, in an unfoldable dataset, values of the observed variables are quadratically (or at least single-peakedly) related to values on the latent variables. The problem, in fact, goes beyond factor analysis and applies more generally to all measurement 
models that assume a linear or monotonic relationship between observed and latent variables, including the (factor-analytic) measurement part of more comprehensive structural equation models [e.g., with the programs LISREL (Jöreskog \& Sörbom, 1988) or EQS (Bentler, 1989)]; classical reliability analysis; and item response theory (including the Rasch model) and its nonparametric analogue, the Mokken model (Mokken \& Lewis, 1982; Sijtsma, Debets, \& Molenaar, 1990).

The difference between the factor model and the unfolding model can be illustrated easily by their mathematical formulations. The factor model is:

$z_{i s}=\sum_{j=1}^{r} b_{i j} \theta_{s j}+\zeta_{i s}$.

The unfolding model is:

$z_{i s}=\sum_{j=1}^{r}\left|\theta_{s j}-\delta_{i j}\right|^{2}+\zeta_{i s}$,

where $z_{i s}$ is the observed value for person $s$ on variable $i$,

$\theta_{s j}$ is the value for person $s$ on factor or latent dimension $j$ (the factor score),

$\delta_{i j}$ is the value of variable $i$ on dimension $j$,

$\zeta_{i s}$ is the value of person $s$ on a unique factor or latent variable that is related to observed variable $i$, and

$b_{i j}$ is the factor loading of variable $i$ on factor $j$.

Note that in the factor model, the higher $\theta_{s j}$ is, the higher $z_{i s}$ will be (a linear relationship). In the unfolding model, in contrast, the greater the Euclidean distance between $\theta_{s j}$ and $\delta_{i j}$, the higher $z_{i s}$ will be (a quadratic relationship). Note also that in factor analysis, recoding the observed scores of a variable (e.g., an item in a rating scale) leads only to a change in the sign of the factor loading for that variable. However, in the unfolding model, changing the data from distances to similarities can lead to serious interpretation difficulties.

The relationship between factor analysis and unfolding is not dependent on the number of factors extracted. However, this paper restricts the discussion to the case of unidimensional bipolar latent constructs, for three reasons. First, most of the relevant substantive discussions in the literature deal with the unidimensional case; for example, the confusion about the dimensionality of political ideology or mood states. Second, most of the diagnostics and solutions here apply only to the unidimensional case. Third, even though some data do not yield a solution with a single latent dimension, the merits of multidimensional unfolding models are still in question. It is suggested that researchers consider instead the applicability of a multiple unidimensional unfolding model (Jacoby, 1991; Van Schuur, 1984, chap. 3).

\section{The Extra Factor Phenomenon in Attitude Data}

Two types of interpretations-substantive and methodological have been proposed for finding orthogonal liberal and conservative political attitude factors (see Krosnick \& Weisberg, 1988, for a summary). According to the substantive interpretation, two independent factors is the correct finding. For example, Weisberg \& Rusk (1970) examined correlations among attitudes toward various political figures and concluded that attitudes were only weakly organized along ideological lines. Weisberg (1980) proposed further that attitudes toward the Democratic and Republican parties in fact do not contrast along a single ideological dimension, but rather represent two evaluative dimensions that are only slightly negatively related.

A methodological explanation was proposed by Green (1988). Green suggested that "... random measurement error will tend to push a strong underlying negative correlation toward, but not past zero" (p. 763). Nonrandom error also may play a role, according to Green: “... survey questions that are worded similarly and placed close together in the interview schedule will evoke similar response biases, and this may give rise to positive error covariance" (p. 763), and this may in turn cause the correlation between the two factors to become slightly positive. In the mood state literature, deficiencies in the wording of questions also have been invoked to explain why unipolar factors were found when bipolar factors were expected (Meddis, 1972).

A related methodological interpretation was proposed by Krosnick \& Weisberg (1988). Following perspective theory (Ostrom \& Upshaw, 1988), Krosnick \& Weisberg suggested that respondents may interpret the end points (anchors) of a scale differently: 
Two respondents who have identical attitudes toward a set of politicians may report those attitudes differently because their perceptions of the meanings of the scale points are different. In particular, one respondent's ratings would be concentrated in one region of the scale, while the other respondent's ratings are concentrated in another region of the scale. Thus, this variation across respondents in terms of perspectives induces a positive correlation between attitude ratings when computed across respondents. (p. 13)

Perspective effects could, suggest Krosnick \& Weisberg (1968), explain the essentially zero correlations between attitudes toward liberal and conservative political issues. When strong, ideologically-based negative correlations between the attitudes are combined with strong positive correlations due to perspective effects, zero correlations could result.

An explanation similar to Krosnick \& Weisberg's (1988) perspective effect has been proposed for results with adjectives. In research on Osgood, Suci, \& Tannenbaum's (1957) semantic differential procedure, Green \& Goldfried (1965) found that when people rated items with two separate adjectives rather than with a pair of antonymous adjectives, factor analysis did not reveal the three expected bipolar factors (evaluation, activity, and potency), but rather a larger number of unipolar factors. Hamilton (1968) and Bentler (1969) found similar unexpected results, and suggested independently that it might be due to response style. If people tend toward acquiescence, they might rate not only one member of a pair of antonymous adjectives as positively related to a particular item, but also its opposite. This idea also has been applied to the study of mood states by Lorr \& Wunderlich (1980). Saris (1988a, 1988b) also independently suggested this interpretation and called it variation in individual response functions to account for systematic errors in magnitude estimations.

In an analysis of affect, Russell (1980) proposed a different methodological explanation for the identification of two unipolar factors when one bipolar factor was expected. He suggested that affective dimensions are related in a systematic fashion that can be represented by a two-dimensional circumplex model. In this model (Guttman, 1954), the factor loadings are the scale values of the stimuli, and they represent the stimuli as points along the circumference of a circle. The model integrates the two-dimensional results of factor analysis with a one-dimensional interpretation by representing each stimulus as the endpoint of a vector through the origin of the space, and assigning the stimulus a scale value equal to the angle of the vector with the horizontal axis. This suggestion also has been applied to the analysis of political emotions (Marcus, 1988).

\section{An Alerintive to Factor Analysis: The Unfoldong Thodel}

There is a more parsimonious explanation than those reviewed above for the conflict between a theoretically expected (unidimensional) outcome and an empirically observed (two-dimensional) result. Factor analysis is an inappropriate mathematical translation of the analyst's assumptions about the structure of a dataset that conforms to the unidimensional unfolding model. This explanation is perhaps most easily illustrated with reference to political ideology. According to Downs' (1957) economic model of democracy, people will prefer political parties, leaders, and pressure groups that are located close to their own position along the liberal-conservative dimension, and they will agree with proposed political measures that are close to their own position. The theoretical model underlying most work on political ideology is therefore better characterized as a distance model than a factor model (see also Brady \& Ansolabehere, 1989; Nygren \& Jones, 1977). According to this model, people can implicitly adopt any position along the liberal-conservative dimension as their ideal point. Political belicf elements also can be assigned a position along the dimension as, of course, can political parties, political leaders, and pressure groups. Such a distance model is known as an unfolding model (Coombs, 1964).

In the literature that attempts to explain the unexpected finding of independent unipolar factors in mood states, Sjöberg, Svensson, \& Persson (1979) came the closest to interpreting their results in terms of the unfolding model. They pointed out that 
Common factor analysis assumes that all items have monotonic (in fact, linear) trace lines. It is reasonable to expect that only items describing the extremes of a mood dimension have such monotonic trace lines. Intermediate items would rather have trace lines taking an inverse U-shape. In terms of correlation statistics, this implies that the covariation between intermediate items will not be well described by linear correlations. (p. 4)

The factor analysis model differs from the unfolding model in that it locates only people and not the stimuli along the latent dimension, and the position the people are assigned has a different meaning. In the unfolding model, people's positions reflect their optimal point along the latent dimension, whereas in factor analysis their positions (i.e., their factor scores) reflect how positive their responses are to the stimuli that maximally represent that factor.

The factor analysis model and the unfolding model make different predictions about the size and the sign of the correlations among the stimuli. This can be illustrated most clearly by reference to a factor-analytic representation with one bipolar factor. According to the factor analysis model, the correlations among all stimuli should be high in absolute value. Correlations among liberal and conservative belief elements (for example) should be negative. However, if the coding order is reversed for one set of stimulus questions, all correlations should become positive.

In the unfolding model, in contrast, only stimuli that are close together along the latent dimension will correlate highly and positively. This is because people tend to give the same responses to both. As the distance between stimuli increases, the correlation between them decreases, often to zero, and then may begin to increase again, this time with the sign reversed to negative (Davison, 1977). A correlation matrix of stimuli that conform to the unidimensional unfolding model, arranged in the order in which they appear along the latent dimension, will show high positive correlations along the diagonal, decreasing correlations downward and to the left, and negative correlations in the lower left-hand corner. The correlation matrix will, therefore, contain both high and low correlations and, counter to predictions of the unidimensional bipolar factor model, it often will be impossible to recode a subset of the stimuli so that all correlations become positive.

Davison (1977) showed that a principal components analysis (PCA) of such a correlation matrix will yield a semicircular, two-factor structure. Variables will be ordered along the semicircle according to their position on the stimulus dimensions. This finding explains why Russell (1980) and Marcus (1988) could interpret their results in terms of a circumplex.

As early as 1960, Coombs \& Kao (1960) conjectured that if data conforming to the unfolding model were subjected to factor analysis, an extra, artificial, constant factor would appear in the solution. They proposed interpreting this "extra dimension" in the factor space as a "social utility" dimension on which individuals have projections inversely related to their average distance from all the other individuals in the unfolding space (Coombs, 1964, p. 191). This hypothesis was mathematically confirmed by Ross \& Cliff (1964) for the case in which data (agreements or preferences) can be regarded as squared distances between people's positions and stimulus positions along the latent dimensions. Their proof is illustrated with an example below.

Coombs (1975) provided another interpretation of the "extra dimension," which he illustrated for the unidimensional case. If items have two attributes that are both desirable (i.e., the more of the attributes the better), a person's preference for one item over the other can be represented as a vector in a twodimensional space. The greater the preference for an item, the greater the distance between the origin of the space and the projection of the item on the person's vector. The cosine of the vector's angle with each of the two attribute dimensions represents the relative importance of the dimensions for the person. If the items also are related to each other such that no item has more of either attribute than any other item, they constitute an efficient set. An efficient set of items can be represented along an arc that is interpretable as a unidimensional subspace in the two-dimensional representation. The point at which the preference vector intersects the arc can be interpreted as the person's ideal point on the arc, and his or her preferences can be specified by un- 
folding the arc at the ideal point. The unidimensional unfolding representation of a set of items is therefore equivalent to the two-dimensional vector representation of that (efficient) set of items. As Coombs pointed out, the argument just given for the two-dimensional vector space can be applied more generally to the vector representation of preferences for an efficient set of items in $r$ dimensions by an $(r-1)$ di-mensional unfolding space.

\section{Consequences of the Extra Factor Phenomenon}

Ross \& Cliff's (1964) proof suggests several additional practical implications of the extra factor phenomenon:

1. The relationship between factor analysis and unfolding is most dramatic in the case of a unidimensional unfolding solution, as in many studies of bipolar concepts. Ross \& Cliff's (1964) proof is more general, however: If the data are representable in a multidimensional unfolding space of $r$ dimensions, a factor analysis solution will give a representation in $r+1$ factors.

2. If the data can be described in terms of more than one unidimensional unfolding scale, each of these scales leads, under factor analysis, to two factors that represent the unipolar halves of what in fact is a single bipolar dimension.

3. If the data cannot be regarded as squared distances, but rather constitute a monotonic transformation of squared distances (e.g., ordinary distances or 5-point ratings on a $\mathbb{L}$ ikert scale), factor analysis will still give $r+1$ rather than $r$ factors, as illustrated in the example presented below.

4. Ross \& Cliff's (1964) proof concerns the eigenvalue/eigenvector decomposition of the covariance matrix rather than the decomposition of the correlation matrix. Because the variables generally do not have the same standard deviation, the factor analysis solution will not show an additional constant factor, but rather a factor that has the highest loadings for the variables that receive the highest values from the respondents.

5. The magnitude of the additional factor's eigenvalue depends on the distribution of persons and stimuli along the latent dimension. Usu- ally it is high, but not always. This means that the extra factor cannot always be identified in the default factor analysis solution, because usually (although this criterion has been questioned) only factors with eigenvalues larger than 1.0 are interpreted. [The reader can verify this easily by constructing an example analogous to the Ross \& Cliff (1964) example below.] Sometimes the eigenvalue of the extra factor is even higher than the eigenvalue(s) of the other factor(s).

6. The factor analysis solution can, in principle, be substantively interpreted if only $r$ (of the $r+1$ ) unrotated factors are used (e.g., Davison, 1977). Which factor should be ignored is not clear from the solution (see 5 above), which hampers substantive interpretation.

7. Ross \& Cliff's (1964) proof is based on a PCA rather than a factor analysis with unique factors. The presence of unique factors in addition to common factors makes it even more problematic to reconstruct the unfolding representation from the factor solution.

\section{Diagnostics for Deciding Petween the Factor Analysis Model and the Unfolding Model}

\section{1: Donble-Centering the Data Matrix}

Consider a data matrix that contains $N$ persons as rows, $n$ stimuli as columns, and preferences by persons for stimuli represented as squared Euclidean distances between person locations and stimulus locations. Ross \& Cliff (1964) showed that $D$ has rank $r+2$ (assuming that both $N$ and $n \geq r+2$ ), where $r$ is the number of dimensions needed to represent persons and stimuli. In the covariance matrix (and hence in the correlation matrix), the appropriate mean column value of is subtracted from each cell value; the rank of this matrix is therefore only $r+1$. This rank implies that the correlation matrix contains only one additional artificial factor. If the data matrix were double-centered (i.e., if both row and column means were 0.0 ), then the resulting matrix would have rank $r$. Ross \& Cliff's diagnostic thus involves establishing the rank of the double-centered data matrix and determin- 
ing whether its rank is equal to the rank of the correlation matrix minus 1. An example is given below. The problem with this diagnostic is that for empirical datasets it does not differentiate between eigenvalues that are low due to random noise and those that are low due to the distribution of persons and stimuli.

\section{2: The Form of the Correlation Matrix}

Davison (1977) showed that if the data conform to a metric unidimensional unfolding model, the correlation matrix of the stimuli, ordered according to their position along the unfolding dimension, forms a simplex pattern; that is, the correlations are highest between adjacent stimuli and decrease monotonically from the diagonal downward and to the left. He warned, however, that a similar simplex pattern can be found if the data conform to Guttman's simplex model. In this case, however, all correlations and all partial correlations will be positive, which is not necessarily true in the unfolding model.

\section{3: Sign of the Partial Correlations}

Davison (1977) also showed that in the unidimensional unfolding model, the sign of the partial correlation of two stimuli with a third depends on the relative position of the three stimuli. A partial correlation $r_{A B C}$ is positive if Stimulus $C$ is located to the left or right of both $A$ and $B$, but it is negative if $C$ is positioned between $A$ and $B$.

Ross \& Cliff's (1964) and Davison's (1977) diagnostics are helpful if the data conform perfectly to the unfolding model. However, for empirical data (e.g., data that are only monotonically related to squared distances) these diagnostics do not always give the desired results. For example, Davison showed a number of simulated and real-life examples in which the partial correlations deviated from the expected patterns. A further drawback is that diagnostics 1 and 3 can only be used if the original data are available.

\section{4: PCA of the Centered Factor Loading Matrix}

For the situation in which only factor loading ma- trices have been reported, Kiers (1986) proposed a procedure for reconstructing the representational space without the extra factor. Kiers proved that if the variables in the data matrix have the same variance, the column-centered transformation of the matrix of factor loadings can be decomposed through a singular value decomposition into a basis for the unfolding space. His proof is summarized in the Appendix.

As a criterion to help evaluate whether empirical data conform to the unfolding model, Kiers used the proportion of the square of the last singular value to the sum of the squares of all singular values of the column-centered matrix of factor loadings. If this proportion is small, the $r+1$ columns of the matrix of factor loadings may contain a constant dimension that should be eliminated.

Unfortunately, this solution does not work perfectly even with perfect unfolding data, because if the data are unfoldable the variables cannot all have the same variance-variables in the center of the dimension will have a lower variance than variables at the ends. However, simulation studies have shown that Kiers' solution is still a useful diagnostic. In particular, the solution works if the data are not standardized; that is, if a PCA is done on the covariance matrix rather than on the correlation matrix.

\section{Summary}

These four diagnostics specify necessary but not sufficient conditions for the unfolding model. Double-centering the data matrix and a PCA of the centered factor loading matrix are useful regardless of the number of factors. The form of the correlation matrix and the signs of the partial correlations are used specifically to determine whether data that conform to a two-factor model also conform to a unidimensional unfolding model.

\section{An Example of Ross \& Chipip's (1964) Proof}

Coombs \& Kao (1960) hypothesized that if data conforming to an unfolding model were analyzed using a factor analytic model, an extra, artificial, constant factor would appear in the solution. This hypothesis then was confirmed by Ross \& Cliff (1964) when the data were squared distances be- 
tween people's positions and stimulus positions along the latent dimensions. This example illustrates this phenomenon.

Figure 1 shows a scale ranging from 1 to 20 with the locations of five stimuli and four persons. The 1D matrix for these data is shown in Table 1 (1) had rank 3). Table 2 shows the centered matrix 1 * (rank 2) and the double-centered matrix ** (rank 1).

Tiguge

A Sample Unfolding Scale for Four Persons (1-4) and Five Stimuli $(\mathrm{A}-\mathrm{E})$

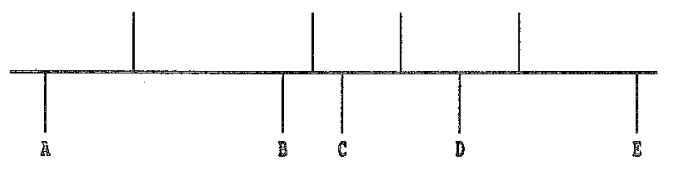

$\begin{array}{lllllllllllllllllllll}0 & 1 & 3 & 3 & 4 & 5 & 6 & 9 & 8 & 9 & 10 & 11 & 12 & 13 & 14 & 15 & 16 & 17 & 18 & 19 & 20\end{array}$

Table 3 is the correlation matrix among the variables from $\mathbb{D}^{*}$. This matrix gave two nonzero eigenvalues: 3.17 and 1.83 . The sign of the correlations was such that it was not possible to recode one or more of the variables to make all correlations positive.

Table 1

The Data Matrix

\begin{tabular}{crrrrr}
\hline & \multicolumn{5}{c}{ Stimulus } \\
\cline { 2 - 6 } Person & A & B & C & D & E \\
\hline 1 & 9 & 25 & 49 & 121 & 289 \\
2 & 81 & 1 & 1 & 25 & 121 \\
3 & 144 & 16 & 4 & 4 & 64 \\
4 & 256 & 64 & 36 & 4 & 16 \\
\hline
\end{tabular}

The original data matrix was recoded into "Likert" format $[(1-4=1)(9-16=2)(25-64=3)$ $(81-169=4)$ (higher $=5)]$; that matrix is shown in Table 4 . The matrix was factor analyzed, and three eigenvalues were larger than $0.0: 2.85,1.92$, and .23 . The first two were much larger than the third. Table 5 shows the factor solutions of the two data matrices. The two factor loading matrices were almost identical, especially when the loadings over $|.5|$ were considered.

\section{Iriplementimg. Uniolonding Amalyses}

One reason why factor analysis. models often are applied to data for which they are inappropriate is that software to implement factor analyses is widely
Table 2

Centered Matrix $\mathbb{D}^{*}$ and Double-Centered Matrix $\mathbb{D} * * *$

\begin{tabular}{|c|c|c|c|c|c|}
\hline \multirow{2}{*}{$\begin{array}{l}\text { Matrix } \\
\text { and } \\
\text { Person }\end{array}$} & \multicolumn{5}{|c|}{ Stimulus } \\
\hline & A & B & $\mathrm{C}$ & $\mathrm{D}$ & $\mathrm{E}$ \\
\hline \multicolumn{6}{|c|}{ Centered Matrix ${ }^{*}($ Rank 2$)$} \\
\hline 1 & -113.5 & -1.5 & 26.5 & 82.5 & 166.5 \\
\hline 2 & -41.5 & -25.5 & -21.5 & -13.5 & -1.5 \\
\hline 3 & 21.5 & -10.5 & -18.5 & -34.5 & -58.5 \\
\hline 4 & 133.5 & 37.5 & 13.5 & -34.5 & -106.5 \\
\hline \multicolumn{6}{|c|}{ Double-Centered Matrix D** (Rank 1) } \\
\hline 1 & -145.6 & -33.6 & -5.6 & 50.4 & 134.4 \\
\hline 2 & -20.8 & -4.8 & -.8 & 7.2 & 19.2 \\
\hline 3 & 41.6 & 9.6 & 1.6 & -14.4 & -38.4 \\
\hline 4 & 124.8 & 28.8 & 4.8 & -43.2 & -115.2 \\
\hline
\end{tabular}

available, whereas software to implement unfolding models is not. Standard statistical packages such as SPSS/PC+ (Norušis, 1986), SAS (SAS Institute, 1985), and BMDP (Dixon, Brown, Engelman, \& Jennrich, 1990) do not currently incorporate unfolding options. Further, most of the unfolding programs that are available [e.g., ALSCAL (Young, Takane, \& Lewyckyj, 1978), KYST (Kruskal, Young, \& Seery, 1973), or Carroll's unfolding programs in Coxon's (1980) $\operatorname{MDS}(\mathrm{X})$ package] do not handle large sample sizes. These programs also often give degenerate results, especially when a unidimensional solution is requested.

Fortunately, several unfolding programs recently have appeared that can handle large sample sizes, explicitly assume an underlying unidimensional latent dimension, and do not degenerate as easily as the earlier programs (Andrich, 1988; Cliff, Collins, Zatkin, Gallipeau, \& McCormick, 1988; DeSarbo \& Hoffman, 1986; Hoijtink, 1990; Poole, 1984; Van Blokland, 1989, 1991; Van Schuur, 1988). Programs for most of these models assume dichotomous (or

Table 3

Correlation Matrix Among the Stimuli from the Centered Matrix ( ${ }^{*}$ )

\begin{tabular}{crrrrr}
\hline \hline & \multicolumn{5}{c}{ Stimuli } \\
\cline { 2 - 6 } Variable & \multicolumn{1}{c}{$\mathrm{A}$} & $\mathrm{B}$ & $\mathrm{C}$ & $\mathrm{D}$ & $\mathrm{E}$ \\
\hline $\mathrm{A}$ & 1.00 & & & & \\
$\mathrm{~B}$ & .71 & 1.00 & & & \\
$\mathrm{C}$ & -.09 & .63 & 1.00 & & \\
$\mathrm{D}$ & -.81 & -.16 & .67 & 1.00 & \\
$\mathrm{E}$ & -.92 & -.37 & .48 & .98 & 1.00 \\
\hline
\end{tabular}


Table 4

Data Matrix Recoded in Likert Format

\begin{tabular}{cccccc}
\hline & \multicolumn{5}{c}{ Stimulus } \\
\cline { 2 - 6 } Person & A & B & C & D & E \\
\hline 1 & 2 & 3 & 3 & 4 & 5 \\
2 & 4 & 1 & 1 & 3 & 4 \\
3 & 4 & 2 & 1 & 1 & 3 \\
4 & 5 & 3 & 3 & 1 & 2 \\
\hline
\end{tabular}

dichotomized) data, although Poole's program assumes squared distances and Van Blokland's assumes complete rank orders, possibly with ties. If dichotomous data conform perfectly to a unidimensional unfolding representation, the data matrix can be permuted in such a way that a parallelogram of $1 \mathrm{~s}$ can be constructed from top left to bottom right. [The unfolding analysis of dichotomous data was therefore termed parallelogram analysis by Coombs (1964).] Recent unfolding models resemble the cumulative scaling models of Guttman (1950), Rasch (1960), and Mokken (1971).

Unfortunately, programs for many of these models are not yet readily available or exist only in experimental versions. An exception is MUDFOLD, a nonparametric unidimensional unfolding program for dichotomous data (Van Schuur \& Post, 1991). Some applications in the field of political ideology are presented in Van Schuur (1984, 1987), an application to marketing research is presented in Van Schuur (1988), and a generalization of the model is presented in Van Schuur (1993a).

MUDFOLD can be used as a diagnostic device to explore whether a maximal subset of unidimensionally unfoldable stimuli can be found in a set of items. The procedure works as follows. If a set of stimuli conforms perfectly to the unidimensional unfolding model, then for each triple of stimuli, ordered according to their position along the unfolding dimension, one response pattern does not occur: the pattern in which the two outer stimuli are coded as 1 (i.e., the person agrees with, is sympathetic to, prefers) but the intervening stimulus is coded as 0 . This pattern (termed the 101-pattern of an ordered triple of stimuli) is therefore called the error pattern for that triple. (Note that this definition of error assumes only an ordinal rating of the stimuli by the respondents.)

In searching for an unfolding scale, MUDFOLD calculates the frequency of the error pattern for each triple in each of its three possible permutations and compares it to the frequency that would have, been expected if responses to the three stimuli were statistically independent. After selecting the best triple as a starting scale, MUDFOLD successively adds further stimuli to it in any position as long as various criteria for unfoldability continue to be met. For a maximal (sub) set of $p$ stimuli to be identified as conforming to an unfolding scale, for each of the $p(p-1)(p-2) / 6$ ordered triples of $p$ stimuli, the frequency of observed error patterns $O$, must be significantly lower than the frequency of expected error patterns $E$ (i.e., error patterns expected under statistical independence). $O$ and $E$ are compared using Loevinger's coefficient of homogeneity, $H$, where $H=1-O / E$.

When data conform perfectly to an unfolding scale, $H=1.0$; when the data are statistically independent, $H=0.0 . H$ can be calculated both for the entire scale and for each individual stimulus in its

Table 5

Unrotated and Varimax Rotated Two-Factor Solutions for the Original Dataset (D) and for D Recoded to Likert Format

\begin{tabular}{|c|c|c|c|c|c|c|c|c|}
\hline \multirow[b]{3}{*}{ Stimulus } & \multicolumn{4}{|c|}{10} & \multicolumn{4}{|c|}{ Likert Format } \\
\hline & \multicolumn{2}{|c|}{ Unrotated } & \multicolumn{2}{|c|}{ Varimax } & \multicolumn{2}{|c|}{ Unrotated } & \multicolumn{2}{|c|}{ Varimax } \\
\hline & 1 & 2 & 1 & 2 & 1 & 2 & 1 & 2 \\
\hline$A$ & -.94 & .35 & -.92 & .38 & -.96 & -.01 & -.94 & -.20 \\
\hline B & -.42 & .91 & -.39 & .92 & .15 & .97 & -.03 & .98 \\
\hline C & .44 & .90 & .47 & .88 & .29 & .93 & .11 & .97 \\
\hline D & .96 & .27 & .97 & .24 & .95 & -.16 & .96 & .02 \\
\hline $\mathrm{E}$ & .99 & .05 & .99 & .01 & .96 & -.29 & .99 & -.11 \\
\hline
\end{tabular}


scale position. In calculating $H$ for a stimulus, the number of observed and expected error patterns are summed over the $(p-1)(p-2) / 2$ triples that contain the stimulus. A stimulus is acceptable in an unfolding scale if its $H_{i}$ value is larger than .30. This criterion alone proves to be almost sufficient for identifying an interpretable unidimensional unfolding scale. The best starting triple of three items, $l, m$, and $n$ should have an overall positive $H_{l m n}$ value above 30 when the three items are in their unfoldable permutation, and a negative $H$ value when the items are in their two other permutations. Additional stimuli will be added only if the $H$ values of each new stimulus and of the newly extended scale as a whole are both sufficiently high, and if new stimuli can be added only in a unique position. For additional criteria used in MUDFOLD, see Van Schuur (1988).

\section{An Lxample: Uniolding American Party} Activists' Atuidudes Toward Political Candidates

In 1980, 697 (Democratic and Republican) party activists from Missouri rated a number of presidential candidates, the Democratic governor, and two state senators on a five-point favorability rating scale (very favorable $=5$, somewhat favorable $=4$, neutral $=3$, somewhat unfavorable $=2$, very unfavorable $=1$ ) (for previous publications using these data see Rapoport, Abramowitz, \& McGlennon, 1986). The unfolding properties of this dataset have been established independently by Böckenholt \& Böckenholt (1990) and Hoijtink (1991).
The correlation matrix among the Likert-scored ratings is given in Table 6 . The correlation matrix contains two sets of high correlations (Carter to Anderson, with values over .40; and Anderson to Reagan, with values generally over .30 ). The correlation matrix was factor analyzed (with the default options) using SPSS/PC+ (Norušis, 1986). The first three eigenvalues were $3.90,2.36, .73$. This indicated a two-factor solution (shown after Varimax rotation in Table 7). Note that the two factors are almost completely unipolar; the only exception to this is the high negative loading for Reagan on the first factor (which was interpreted as a "Democratic" factor).

\section{Resulis From the Four Diagnostics for Determining Whether artaset is Unfoldable}

Double-centering the data matrix. To find the highest eigenvalues of the double-centered data matrix, the row mean score for each person was subtracted from his or her score for each of the nine candidates, and then the rank of the correlation matrix of the new variables was determined. The first four eigenvalues then were $3.72,1.56,1.08$, and .77, which may be interpreted as a matrix not of rank 1 . Because the second and third eigenvalues were still high, this criterion did not indicate whether the data formed a unidimensional unfolding scale. Because the differences are so small, this criterion does not indicate clearly whether the data form an unfolding scale.

The form of the correlation matrix. The correlation matrix (Table 6) shows the simplex pattern,

Toble 6

Correlation Matrix of Favorability Ratings for Nine Candidates by 697 Party Activists From Missouri in 1980

\begin{tabular}{lrrrrrrrrr}
\hline & \multicolumn{1}{c}{ Candidate } \\
\cline { 2 - 9 } Candidate & $\mathrm{C}$ & $\mathrm{DS}$ & $\mathrm{G}$ & $\mathrm{K}$ & $\mathrm{Br}$ & $\mathrm{A}$ & $\mathrm{Bu}$ & $\mathrm{RS}$ & $\mathrm{R}$ \\
\hline Carter (C) & 1.00 & & & & & & & \\
Democratic Senator (DS) & .72 & 1.00 & & & & & & & \\
Governor (G) & .57 & .72 & 1.00 & & & & & & \\
Kennedy (K) & .43 & .58 & .55 & 1.00 & & & & & \\
Brown (Br) & .40 & .47 & .49 & .74 & 1.00 & & & & \\
Anderson (A) & .35 & .45 & .41 & .56 & .60 & 1.00 & & & \\
Bush (Bu) & -.04 & -.11 & .01 & .14 & .34 & .44 & 1.00 & & \\
Republican Senator (RS) & -.02 & .09 & .12 & .17 & .29 & .36 & .48 & 1.00 & \\
Reagan (R) & -.49 & -.59 & -.34 & -.19 & .04 & .02 & .57 & .33 & 1.00 \\
\hline
\end{tabular}


Table 7

Varimax Factor Loadings of the Nine Candidates

\begin{tabular}{lcc}
\hline Candidate & Factor 1 & Factor 2 \\
\hline Carter (C) & .80 & -.15 \\
Democratic Senator (DS) & .91 & -.15 \\
Govemor (G) & .81 & .01 \\
Kennedy (K) & .77 & .27 \\
Brown (Br) & .67 & .50 \\
Anderson (A) & .60 & .56 \\
Bush (Bu) & -.02 & .86 \\
Republican Senator (RS) & .12 & .69 \\
Reagan (R) & -.53 & .72 \\
\hline
\end{tabular}

with correlations that are both high and low and both positive and negative. In general, correlations are highest around the diagonal and decrease downward and to the left. Some correlations between Democratic (Carter, Kennedy, and Brown) and Republican (Bush and Reagan) candidates were negative, but not all, so it is not possible to simply reverse the favorability codings for one set of candidates so that all correlations become positive. This criterion, therefore, suggests that the data may be represented as a unidimensional unfolding scale.

Sign of partial correlations. The criterion of examining the partial correlations can only be used for a hypothesized order in which the items might be (unidimensionally) unfoldable. Such an order can be derived from the order in which the correlation matrix of the items shows a simplex pattern or from the results of an unfolding analysis. The partial correlations [using SPSSX (SPSS, Inc., 1986)] gave somewhat mixed support for the unidimensional unfolding solution reported below. Given nine stimuli, the signs of $252(9 \times 8 \times 7 / 2)$ partial correlations must be considered. 40 of these, or $16 \%$, had the wrong sign. Most of these (33) should have been negative but were in fact positive. The candidates contributing to the highest number of partial correlations with the incorrect sign were Carter ( 13 cases) and the Democratic senator (11); Reagan contributed the least (5).

PCA of the centered factor loading matrix. The first singular value of the centered factor loading matrix was 1.68 , and the second was .55 . The value for Kiers' criterion was, therefore, .10 , which is rather small. This criterion suggested a unidimensional unfolding solution (as did the second diagnostic, the form of the correlation matrix).

\section{Unfololding Analysis}

The favorability ratings of the party activists were first dichotomized (very favorable versus the remaining scores), and these data were analyzed by MUDFOLD. It revealed a unidimensional unfolding representation of the candidates (see Table 8). The coefficient of homogeneity for this unfolding scale was very high $(H=.85)$, which indicated that the data were well represented by a single unfold. ing dimension.

Once an unfolding scale has been found, it is possible to assign scale values to persons and to reconstruct the item response functions. The method for assigning scale values is explained elsewhere (Van Schuur, 1993a); the lowest possible value is 1 and the highest is 17 . On the basis of the frequency distribution of the scale values, adjacent scale value groups with approximately equal numbers of respondents can be formed. Low and high values indicate a preference for Democratic and Republican candidates, respectively. Table 9 gives the percentage of respondents in each scale value group who gave a very favorable response to each of the candidates. For example, starting from the bottom of the second column, 54 respondents did not give any very favorable responses, 224 were very favorable only to Reagan, and 76 were very favorable only to Reagan and the Republican senator.

If the unfolding model holds, a single-peaked dis-

Table 8

Proportion of Respondents Who Rated Their Attitude Toward a Candidate as Very Favorable $(p)$ and the Scalability Coefficient of Each Stimulus $\left(H_{i}\right)$ From a MUDFOLD Analysis of Ratings of Nine Candidates

\begin{tabular}{lcc}
\hline \hline Candidate & $p_{i}$ & $H_{i}$ \\
\hline Carter (C) & .24 & .91 \\
Democratic Senator (DS) & .32 & .83 \\
Governor (G) & .18 & .82 \\
Kennedy (K) & .08 & .82 \\
Brown (Br) & .01 & .82 \\
Anderson (A) & .02 & .80 \\
Bush (Bu) & .07 & 82 \\
Republican Senator (RS) & .19 & .57 \\
Reagan (R) & .49 & .96 \\
\hline
\end{tabular}


Table 9

Percentage of Respondents Within Each Scale Value Group Who Gave a Very Favorable Response to Each of the Nine Candidates

\begin{tabular}{lrrrrrrrrrrr}
\hline Scale & \multicolumn{1}{c}{ Value } & $N$ & $\mathrm{C}$ & $\mathrm{DS}$ & $\mathrm{G}$ & $\mathrm{K}$ & $\mathrm{Br}$ & $\mathrm{A}$ & $\mathrm{Bu}$ & $\mathrm{RS}$ & $\mathrm{R}$ \\
\hline $1-2$ & 79 & 100 & 71 & 0 & 0 & 0 & 0 & 0 & 0 & 0 \\
3 & 107 & 74 & 94 & 66 & 2 & 0 & 1 & 0 & 5 & 0 \\
$4-7$ & 82 & 11 & 70 & 66 & 61 & 6 & 6 & 0 & 13 & 0 \\
$8-15$ & 75 & 0 & 17 & 4 & 7 & 4 & 15 & 64 & 51 & 57 \\
16 & 76 & 0 & 0 & 0 & 0 & 0 & 0 & 0 & 100 & 100 \\
17 & 224 & 0 & 0 & 0 & 0 & 0 & 0 & 0 & 0 & 100 \\
Missing & 54 & & & & & & & & & \\
\hline
\end{tabular}

tribution both rowwise and columwise, with the peak moving from top left to bottom right, would be expected in the cells of this table. This pattern is clearly visible. The few exceptions involve the governor $(G)$ and the senators (DS and RS), which suggests that their very favorable ratings might have been based on an additional (state) criterion.

\section{Conclusions}

The inappropriate use of factor analysis for data that conform to the unfolding model is widespread, due to the incorrect belief that data collected in a certain way (in this case, Likert items or rating scales) necessarily calls for a specific form of data analysis. Ross \& Cliff (1964) provided a formal proof that established that when data that conform to an unfolding representation in $r$ dimensions are factor analyzed, a representation in $r+1$ factors will result. Ross \& Cliff's proof was limited to the case in which the data consisted of squared distances. However, as shown here, both in a simulated example (the Ross \& Cliff example above) and in a substantive political ratings example, data consisting of monotonic transformations of squared distances-which is how Likert-items or rating scales usually are interpreted-can give rise to the same phenomenon.

The extra factor phenomenon is most dramatic when the unfolding representation is unidimensional, because the two-factor solution treats what in fact are the two halves of the unfolding dimension as independent. Stated in general terms, the problem arises from using an inappropriate measurement model. A model that assumes a quadratic (or at least single- peaked) relationship between a latent dimension and the indicators designed to measure it is needed, but instead a model that assumes a linear (or at least monotonically increasing) relationship is used.

The extra factor phenomenon may have affected theory development in a number of substantive areas in the social sciences. Two areas in which two independent factors or traits have been accepted when a one-dimensional bipolar latent dimension might be expected include work satisfaction and androgyny research. In research on work satisfaction, Herzberg, Mausner, \& Snyderman (1959) suggested that overall job satisfaction depends on two sets of indepen dent factors: positive factors, called motivator factors or satisfiers, and negative factors, termed hygiene factors or dissatisfiers. Their theory was criticized by House \& Wigdor (1967) and Hinrichs \& Mischkind (1967) who were surprised that the two factors were independent. With respect to psychological androgyny, it has been suggested that masculinity and femininity are independent dimensions (e.g., Bem, 1974, 1981; Pedhazur \& Tetenbaum, 1979). Unfolding analyses of work satisfaction data collected in the European values study (Harding, Phillips, \& Fogarty, 1986) and of Dutch androgyny data (Van Schuur, 1993b) suggest that in both cases the two independent dimensions can be represented as the bipolar halves of one unfolding dimension.

\section{Appendin: Kiers' Sollotion}

An eigenvalue/eigenvector decomposition of $\mathrm{D}_{c}{ }^{\mathrm{D}} \mathrm{D}$ can be written as

$\mathbb{D O}_{c}^{\prime} \mathbb{D}_{c}=\mathbf{K}^{2} \mathbf{K}^{\prime}$ 
in which

$\mathrm{K}^{\prime} \mathrm{K}_{\mathrm{K}}=\mathrm{K}_{\mathrm{K}} \mathrm{K}^{\prime}=\mathrm{I}$,

Where $K$ is an orthonormal matrix and $\Lambda$ is a diagonal matrix with the eigenvalues of $\mathbb{D}_{c}^{\prime} \mathbb{D}_{c}$ on the diagonal. The matrix of factor loadings and the matrix of factor scores $\mathbb{T}$ are given by $\mathbb{S}=\mathbb{K} \Lambda$ and $\mathrm{T}=\mathrm{D}_{c} \mathrm{~K}^{-1}$.

For a complete principal components solution it now holds that

$\mathrm{D}_{\mathrm{c}}=\mathrm{DK}_{\mathrm{K}} \Lambda^{-1} \Lambda \mathrm{K}^{\prime}=\mathrm{TS}^{\prime}$.

From Ross \& Cliff (1964)

$\mathbb{D}_{c}=-2 \mathbb{A}_{c} \mathbb{P}_{c}$

was derived. Therefore,

$-2 \mathbf{H}_{c}^{\prime}=\mathbf{S} \mathrm{P}^{\prime}$

or

$S=-2 \mathbb{A}_{c}^{\prime} \mathbb{E}\left(\mathbb{F}^{\prime} \mathbb{F}^{-1}\right.$.

Define

$\mathrm{T}=-2 \mathbf{A}_{c}^{\prime}\left(\mathrm{F}^{\mathrm{T}} \mathrm{T}^{-1}\right.$

so that $\mathrm{S}=\mathrm{R}_{c} \mathrm{~T}$, where $\mathrm{T}$ is nonsingular and $\mathrm{R}$ is an $n \times(r+1)$ matrix containing the stimulus configuration in the first $r$ columns and a last column of $-1 \mathrm{~s}$.

The last column of can be removed by column-centering $\mathbb{\$}$ :

$\mathbb{S}_{C}=(\tilde{B}: 0) \mathrm{T}$,

where $\mathbb{S}_{c}$ is the column-centered matrix of factor loadings, and $\tilde{H}$ is the centered stimulus configuration. Therefore, $S_{c}$ and $\tilde{T}_{\text {T }}$ represent the same space. The singular value decomposition in

$S_{c}=\mathbb{P}_{r} Q_{r}$

gives $\mathbb{P}_{r}$ as a basis for the stimulus configuration.

\section{References}

Andrich, D. (1988). The application of an unfolding model of the PIRT type to the measurement of attitude. Applied Psychological Measurement, 12, $33-51$.

Bem, S. L. (1974). The measurement of psychological androgyny. Journal of Consulting and Clinical Psy- chology, 42, 115-162.

Bem, S. L. (1981). The BSRI and gender schema theory: A reply to Spence and Helmreich. Psychological Review, 88, 369-371.

Bentler, P. M. (1969). Semantic space is (approximately) bipolar. The Journal of Psychology, 71, 33-40.

Bentler, P. M. (1989). EQS structural equations program manual. Los Angeles CA: BMDP Statistical Software.

Böckenholt, U., \& Böckenholt, I. (1990). Modeling individual differences in unfolding preference data: $A$ restricted latent class approach. Applied Psychological Measurement, 14, 256-269.

Brady, H. E., \& Ansolabehere, S. (1989). The nature of utility functions in mass publics. American Political Science Review, 83, 143-163.

Cliff, N., Collins, L. M., Zatkin, J., Gallipeau, D., \& McCormick, D. J. (1988). An ordinal scaling method for questionnaire and other ordinal data. Applied Psychological Measurement, 12, 83-97.

Conover, P. J., \& Feldman, S. (1981). The origins and meaning of liberal-conservative self-identifications. American Joumal of Political Science, 25, 617-645.

Coombs, C. H. (1964). A theory of data. New York: Wiley.

Coombs, C. H. (1975). A note on the relation between the vector model and the unfolding model for preferences. Psychometrika, 40, 115-116.

Coombs, C. H., \& Kao, R. C. (1960). On a connection between factor analysis and multidimensional unfolding. Psychometrika, 25, 219-231.

Coxon, A. P. M. (1980), MDS(X) user's manual (SV3). Edinburgh: University of Edinburgh, Program Library Unit.

Davison, M. L. (1977). On a metric, unidimensional unfolding model for attitudinal and developmental data. Psychometrika, 42, 523-548.

DeSarbo, W. S., \& Hoffman, D. L. (1986). Simple weighted threshold models for the spatial representation of binary choice data. Applied Psychological Measurement, 10, 247-264.

Diener, E., \& Emmons, R. A. (1985). The independence of positive and negative affect. Journal of Personality and Social Psychology, 47, 1105-1117.

Dixon, W. J., Brown, M. B., Engelman, L., \& Jennrich, R. I. (1990). BMDP: Statistical software mamal (Vol. I and II; revised edition). Berkeley: University of California Press.

Downs, A. (1957). An economic theory of democracy. New York: Harper and Row.

Green, D. P. (1988). On the dimensionality of public sentiment toward partisan and ideological groups. American Journal of Political Science, 32, 758-780.

Green, R. F., \& Goldfried, M. R. (1965). On the bipolarity of semantic space. Psychological Monographs: General and Applied, 79 (6), 1-31. 
Guttman, L. (1950). The basis for scalogram analysis. In S. A. Stouffer, L. Guttman, E. A. Suchman, P. F. Lazarsfeld, S. A. Star, \& J. A. Clausen, Measurement and prediction: Studies in social psychology in World War II (Vol. IV; pp. 60-90). Princeton NJ: Princeton University Press.

Guttman, L. (1954). A new approach to factor analysis: The radex. In P. R. Lazarsfeld (Ed.), Mathematical thinking in the social sciences (pp. 258-348). Glencoe IL: Free Press.

Hamilton, D. (1968). Personality attributes associated with extreme response style. Psychological Bulletin, 69, 192-203.

Harding, S., Phillips, D., \& Fogarty, M. (1986). Contrasting values in Western Europe: Unity, diversity \& change. London: Macmillan.

Herzberg, F., Mausner, B., \& Snyderman, B. B. (1959). Motivation to work. New York: Wiley.

Hinrichs, J. R., \& Mischkind, L. A. (1967). Empirical and theoretical limitations of the two-factor hypothesis of job satisfaction. Journal of Applied Psychology, 5I, 191-200.

Hoijtink, H. (1990). A latent trait model for dichotomous choice items. Psychometrika, 55, 641-656.

Hoijtink, H. (1991). PARELLA. Measurement of latent traits by proximity items. Leiden, The Netherlands: DSWO Press (Methodology Series No. 20).

House, R. J., \& Wigdor, L. A. (1967). Herzberg's dualfactor theory of job satisfaction and motivation: A review of the evidence and a criticism. Personnel Psychology, 20, 369-389.

Jacoby, W. (1991). Data theory and dimensional analysis. Newbury Park CA: Sage.

Jöreskog, K. G., \& Sörbom, D. (1988). LISREL VII: A guide to the program and applications. Chicago: SPSS, Inc.

K.iers, H. (1986). Meerdimensionale ontvouwing met behulp van principale componenten analyse (Mulidimensional unfolding with the help of principal components analysis). Unpublished report, University of Groningen, The Netherlands.

Krosnick, J. A., \& Weisberg, H. F. (1988, August). Liberal/conservative ideological structures in the mass public: A study of attitudes toward politicians and social groups. Paper presented at the annual meeting of the American Political Science Association, Washington D.C.

Kruskal, J. B., Young, F. W., \& Seery, J. B. (1973). How to use KYST, a very flexible program to do multidimensional scaling and unfolding. Murray Hill NJ: Bell Laboratories.

Lor, M., \& Shea, T. M. (1979). Are mood states bipolar? Joumal of Personality Assessment, 43, 468-472.

Lorr, M., \& Wunderlich, R. A. (1980). Mood states and acquiescence. Psychological Reports, 46, 191-195.
Marcus, G. E. (1988). The structure of emotional response: 1984 presidential elections. American Political Science Review, 82, 737-761.

Meddis, R. (1972). Bipolar factors in mood adjective checklists. British Journal of Social and Clinical Psychology, 11, 178-184.

Mokken, R. J. (1971). A theory and procedure of scale analysis. New York: De Gruyter (Mouton).

Mokken, R. J., \& Lewis, C. (1982). A nonparametric approach to the analysis of dichotomous item responses. Applied Psychological Measurement, 10 , 417-430.

Norušis, M. (1986). SPSS/PC+ for the IBM PC/XT/AT. Chicago: SPSS Inc.

Nowlis, V., \& Nowlis, H. (1956). The description and analysis of mood. Annals of the New York Academy of Sciences, 55, 345-355.

Nygren, T. E., \& Jones, L. E. (1977). Individual differences in perceptions and preferences for political candidates. Journal of Experimental and Social Psychology, 13, 182-197.

Osgood, C. E., Suci, G. J., \& Tannenbaum, P. H. (1957). The measurement of meaning. Urbana: University of Illinois Press.

Ostrom, T. M., \& Upshaw, H. S. (1968). Psychological perspective and attitude change. In A. G. Greenwald, T. C. Brock, \& T. M. Ostrom (Eds.), Psychological foundations of attitudes (pp. 217-242). New York: Academic Press.

Pedhazur, E. J., \& Tetenbaum, T. J. (1979). Bem Sex Role Inventory: A theoretical and methodological cri\{ique. Joumal of Personality and Social Psychology, 37, 996-1016.

Poole, K. T. (1984), Least squares metric, unidimensional unfolding. Psychometrika, 49, 311-323.

Rapoport, R. B., Abramowitz, A. I., \& McGlennon, J. (1986). The life of the parties. Activists in presidential politics. Lexington: The University of Kentucky Press.

Rasch, G. (1960). Probabilistic models for some intelligence and attainment tests. Copenhagen: Danish Institute for Educational Research. [Expanded edition, University of Chicago Press, 1980.]

Ross, J., \& Cliff, N. (1964). A generalization of the interpoint distance model. Psychometrika, 29, $167-176$.

Russell, J. A. (1980). A circumplex model of affect. Journal of Personality and Social Psychology, 39, 1161-1178.

Saris, W. E. (1988a). Individual response functions. In W. E. Saris \& I. N. Gallhofer (Eds.), Sociometric research: Data collection and scaling (Vol. 1) (pp. 200-214). London: MacMillan.

Saris, W. E. (1988b). Variation in response functions. A source of measurement error in attitude research. 
Amsterdam, The Netherlands: Sociometric Research Foundation.

SAS Institute, Inc. (1985). SAS user 's guide: Basics. Cary NC: Author.

Sijtsma, K. P. D., \& Molenaar, I. W. (1990). Mokken scale analysis for polychotomous items: Theory, a computer program and an empirical application. Quality and Quantity, 24, 173-188.

Sjöberg, L., Svensson, E., \& Persson, L.-O. (1979). The measurement of mood. Scandinavian. Journal of Psychology, 20,1-18.

SPSS, Inc. (1986). SPSSX user's guide (2nd edition). New York; McGraw-Hill.

Van Blokland, A. W. (1989). Unfolding and consensus ranking: A prestige ladder for technical occupations. In G. de Soete, H. Feger, \& K. C. Klauer (Eds.), New developments in psychological choice modeling (pp. 237-258). Amsterdam, The Netherlands: North-Holland.

Van Blokland, R. (1991). Unfolding and consensus ranking for individual preferences. Leiden, The Netherlands: DSWO Press (Methodology Series, No. 18).

Van Schuur, H. (1984). Structure in political beliefs. Amsterdam, The Netherlands: CT Press.

Van Schuur, W. H. (1987). Constraints in European party activists' sympathy scores for interest groups: The leftright dimension as dominant structuring principle. European Journal of Political Research, 15, 347-362.

Van Schuur, W. H. (1988). Stochastic unfolding. In W. E. Saris \& 1. N. Gallhofer (Eds.), Sociometric research: Data collection and scaling (Vol. 1; pp. 137-158). London: MacMillan.

Van Schuur, W. H. (1993a). Nonparametric unidimensional unfolding for multicategory data. Political Analysis, 4, $41-74$.
Van Schuur, W. H. (1993b). Masculinity and femininityor androgyny? A comparison of three unfolding models. In H. Oud \& R. A. W. Vogelesang (Eds.), Advances in longitudinal and multivariate analysis in the behavioral sciences (pp. 219-233). Proceedings of the SMABS conference 1992 in Nijmegen, Holland. Nijmegen: Institut voor Toegepaste Sociale Wetenschappen.

Van Schuur, W. H., \& Post, W. (1991). User's manual for MUDFOLD, a program for multiple unidimensional unfolding. Groningen, The Netherlands: ProGamma.

Weisberg, H. F. (1980). A multidimensional conceptualization of party identification. Political Behavior, 2. 33-60.

Weisberg, H. F., \& Rusk, J. G. (1970). Dimensions of candidate evaluation. American Political Science Review. 64, 1167-1185.

Young, F. W., Takane, Y., \& Lewyckyj, R. (1978). ALSCAL: A nonmetric multidimensional scaling program with several difference options. Behavior Research Methods and Instrumentation, 10, 451-453.

\section{Ack}

The authors thank Melissa Bowerman and Tom Snijders for their comments on an earlier version of this paper, and Ron Rapoport for sharing his data.

\section{A}

Send requests for reprints or further information to $W$. H. van Schuur, Department of Sociology, University of Groningen, Grote Rozenstraat 31,9712 TG Groningen, The Netherlands. Internet: h.van.schuur@ppsw.rug.nl. 